\title{
O AUMENTO DO CONTINGENTE POPULACIONAL DE IDOSOS NO BRASIL E A ATENÇÃo PRIMÁRIA À SAÚDE: UMA REVISÃO DE LITERATURA
}

\author{
Kaio Keomma Aires Silva Medeiros ${ }^{1}$ \\ Alexsandro Silva Coura ${ }^{2}$ \\ Rayanne Tavares Ferreira ${ }^{3}$
}

MEDEIROS, K. K. A. S.; COURA, A. S.; FERREIRA, R. T. O aumento do contingente populacional de idosos no Brasil e a atenção primária à saúde: uma revisão de literatura. Arq. Cienc. Saúde UNIPAR, Umuarama, v. 21, n. 3, p, 201-207, set./dez. 2017.

\begin{abstract}
RESUMO: Objetivou-se discutir o aumento do contingente populacional de idosos no Brasil e a sua repercussão na Atenção Primária à Saúde. Revisão crítica da literatura científica, realizada na Biblioteca Virtual de Saúde, a partir dos descritores controlados "Atenção Primária à Saúde", "Saúde do Idoso" e "Envelhecimento", considerando registros bibliográficos em português e em inglês. Procederam-se dois estágios de revisão, compreendendo, no primeiro, a imersão ou acesso a literatura e, no segundo, a formulação de eixos de discussão ou agrupamento de textos por temas. Os resultados contemplaram 38 referências, de diferentes tipologias, incluindo: $63 \%$ de artigos, $11 \%$ de leis e portarias, $26 \%$ de publicações e recomendações de organismos nacionais e internacionais, a partir das quais se conformaram quatro eixos gerais de discussão, contemplando as temáticas: aspectos demográficos, impactos na saúde, atenção primária e avaliação em saúde. Concluiu-se que países como o Brasil têm experimentado um aumento populacional de idosos, sem uma adequada preparação para esta realidade. A Saúde Pública é um setor sobremaneira afetado, sobretudo pelo aumento de doenças crônicas, hospitalização e a dependência, comuns entre aqueles indivíduos. A Atenção Primária em Saúde tem um papel fundamental na assistência ao emergente grupo de idosos, mas ações devem ser empreendidas para fortalecê-la, destacando a criação e manutenção de mecanismos de avaliação.
\end{abstract}

PALAVRAS-CHAVE: Atenção Primária à Saúde. Envelhecimento. Saúde do Idoso.

\section{THE INCREASE IN THE ELDERLY POPULATION IN BRAZIL AND ITS EFFECT ON PRIMARY HEALTH CARE: A LITERATURE REVIEW}

\begin{abstract}
The aim of this study was to discuss the increase in the elderly population in Brazil and its impact on primary health care. A critical review of the scientific literature was carried out in the Virtual Health Library using "Primary Health Care", "Health of the Elderly" and "Aging" as descriptors (both in Portuguese and in English). The review was carried out in two stages, the first one comprising the immersion or access to literature and the second stage comprised the formulation of discussion axes or grouping of texts per topic. The results included 38 references of different typologies, including $63 \%$ articles, $11 \%$ laws and ordinances, $26 \%$ publications and recommendations from both national and international organizations. From those, four discussion axes were designed, which encompassed demographic aspects, health impacts, primary care and health assessment. It could be concluded that countries such as Brazil have experienced an increase in the elderly population without an adequate preparation for such reality. Public Health is extremely affected, mainly due to the increase in chronic diseases, hospitalization and dependence, common among those individuals. Primary Health Care plays a fundamental role in assisting the emerging elderly group, but actions must be taken in order to strengthen it, highlighting the creation and maintenance of assessment mechanisms.
\end{abstract}

KEYWORDS: Aging. Elderly. Primary Health Care.

\section{Introdução}

O aumento da expectativa de vida das pessoas sempre foi uma aspiração das sociedades (VERAS, 2009). De fato, uma realidade, o resultado mais evidente deste fenômeno é a elevação, em termos absolutos e relativos, do quantitativo populacional de idosos, quer no Brasil, quer em outros países (ARAÚJO; POÚL; MARTINS, 2011; BORGES; CAMPOS; SILVA, 2015).

Para além das alterações no quadro demográfico, entretanto, viver mais impeliu importantes modificações no panorama epidemiológico, particularmente em virtude do aumento exponencial das Doenças e Agravos Não Transmissíveis (DANT), sobretudo àquelas de natureza crônico-degenerativa, mais comuns em idosos e que tem sido responsáveis pela maioria das internações e mortes. E, ainda, um conjunto de realidades, que permeiam a sua inserção na sociedade moderna, as quais podem, inclusive, contribuir para o surgimento ou agravamento daquelas, fazendo-os demandar, naturalmente, maior atenção, inclusive não restrita ao setor saúde, quando comparado com indivíduos de outros grupos populacionais de adultos (DANTAS et al., 2017).

As realidades supracitadas, quer aquelas que dizem respeito ao adoecimento, quer as que se referem à condição e inserção do idoso na modernidade, seguramente afetam o Sistema Único de Saúde (SUS), e, muito particularmente, à Atenção Primária à Saúde (APS). Enquanto seu nível reorganizador, a APS tem potencial para responder às novas demandas assistenciais dos idosos, dada a sua inequívoca importância estratégica, no âmbito da promoção e prevenção em saúde, assim como no tratamento e reabilitação, e, ainda, quando bem estruturada, dar vazão a até $80 \%$ dos problemas e necessidades que levam um indivíduo a procurar um serviço de saúde (CARNEIRO, 2014; ONOCKO-CAMPOS et al., 2014).

Não obstante, para que possa, de fato, responder as

DOI: $10.25110 /$ arqsaude.v21i3.2017.6034

${ }^{1}$ Faculdade de Saúde Pública da Universidade de São Paulo. Rua Francisco Calixto, 111, Monte Santo, Campina Grande/PB, Brasil, CEP: 58400750. e-Mail: keomma.kaio@gmail.com

${ }^{2}$ Rua Vigário Calixto, 1369, Catolé. CEP: 58410340. e-Mail: alexcoura_@hotmail.com. Programa de Pós-Graduação em Saúde Pública da Universidade Estadual da Paraíba.

${ }^{3}$ Departamento de Enfermagem da Universidade Estadual da Paraíba. Rua Espírito Santo, 92, Liberdade, Campina Grande/PB, Brasil, CEP: 584014030. e-Mail: rayanneetf@gmail.com 
demandas de atenção que acompanham o aumento da longevidade e, por conseguinte, a elevação no quantitativo populacional de idosos, é preciso que a APS esteja suficientemente alinhada com os atributos que a identificam e a tornam única entre os outros níveis de atenção de maior densidade tecnológica (porta de entrada, longitudinalidade, integralidade e coordenação do cuidado) (STARFIELD, 2002). E, ainda, preparada para lidar com as novas realidades demográficas e epidemiológicas, conforme mencionadas (DANTAS et al., 2017), que têm contribuído para uma série de insidiosos e persistentes problemas de Saúde Pública, razões pelas quais problemáticas como esta precisam ser debatidas, na perspectiva de subsidiar mudanças.

Refletindo acerca das questões ora expostas e na tentativa de oferecer, além de um contributo à literatura geral da Saúde Pública, que tem demandado estudos nas temáticas aqui abordadas, conforme se percebe na prática acadêmica e assistencial; e, adicionalmente, um incremento em discussão tão atual, de que a consolidação do SUS não pode prescindir do desenvolvimento da APS, e esta, por sua vez, da crescente massa populacional de idosos e situações circunscritas, que tem demandado maior atenção, questionou-se: Qual o panorama do aumento do contingente populacional de idosos no Brasil e quais são as suas repercussões na Saúde Pública, particularmente, na Atenção Primária à Saúde?

Por tudo, objetivou-se discutir o aumento do contingente populacional de idosos no Brasil e a sua repercussão na atenção primária a saúde.

\section{Material e Métodos}

O presente estudo trata-se de uma revisão crítica da literatura científica, realizado na Biblioteca Virtual de Saúde, com referências publicadas até 2015.

A partir dos descritores controlados "Atenção Primária à Saúde"; "Saúde do Idoso"; e "Envelhecimento", utilizados durante a pesquisa eletrônica, foram consideradas 38 referências, nos idiomas inglês ou português, de diferentes tipologias: $63 \%$ de artigos $(n=24), 11 \%$ de leis e portarias $(n=4), 26 \%$ de publicações e recomendações de organismos nacionais e internacionais $(n=10)$, a partir dos quais se procedeu à revisão.

A fim de apreender mais profundamente a complexidade entre as temáticas 'idoso'e 'atenção primária a saúde’, as quais compõem, em conjunto, o objeto central deste trabalho, procederam-se dois estágios de revisão, a saber: imersão e formulação de eixos de discussão. O primeiro compreendeu o acesso e leitura, inicialmente de títulos e resumos, dos constructos encontrados, optando por aqueles com potencial para integrar o corpus de textos a serem lidos integralmente e analisados; e, no segundo, formulou-se um quadro que pudesse compilar, a partir da semântica, ou agrupamento por temas, assuntos que emergiram na revisão (Tabela 1).
Tabela 1: Apresentação e descrição dos eixos de discussão

\begin{tabular}{c|l}
\hline \multicolumn{1}{c|}{$\begin{array}{c}\text { EIXOS DE } \\
\text { DISCUSSÃO }\end{array}$} & \multicolumn{1}{c}{ ABORDAGEM } \\
\hline Aspectos demográficos & $\begin{array}{l}\text { O aumento do contingente } \\
\text { populacional de idosos no Brasil }\end{array}$ \\
\hline Impactos na Saúde & $\begin{array}{l}\text { Elevação da população de idosos } \\
\text { e sua repercussão na Saúde } \\
\text { Pública }\end{array}$ \\
\hline Atenção primária & $\begin{array}{l}\text { Atenção primária à saúde e o } \\
\text { fortalecimento da assistência em } \\
\text { saúde do idoso }\end{array}$ \\
\hline Avaliação em saúde & $\begin{array}{l}\text { Avaliação da atenção primária } \\
\text { a saúde para qualificar a } \\
\text { assistência em saúde do idoso }\end{array}$ \\
\hline
\end{tabular}

Fonte: Os autores

Ressalta-se, porém, que em virtude das características deste estudo, centrado na revisão narrativa das temáticas em pauta, não houve a pretensão de compilar uma estrutura rígida, quer na etapa de imersão, quer na de formulação de eixos de discussão, mas antes, um direcionador do trabalho de revisão, de modo a facilitar uma interação significativa entre o pesquisador com o material coletado, e favorecer a construção de resultados e discussões mais estruturados.

\section{Resultados e Discussão}

\section{O aumento do contingente populacional de idosos no Brasil}

A discussão sobre o aumento do contingente populacional de idosos, não raras vezes, tem sido matizada e, se por um lado apresenta-se como um dos principais legados do processo de modernização das sociedades, do outro, é referido como uma fonte inequívoca de preocupações políticas, econômicas, e, sobretudo, sociais e de saúde; sendo, portanto, forçoso, que as sociedades discutam essa questão, para que cientes do cenário presente melhor planejem o futuro (BORGES; CAMPOS; SILVA, 2015; MIRANDA; MENDES; SILVA, 2016; PIUVEZAM, 2016).

De todo modo, é certo dizer que em virtude de múltiplos e variados fatores, entre eles e, talvez, principalmente, a redução das taxas de mortalidade, fecundidade e natalidade, tem havido uma persistência na transição demográfica - fenômeno caracterizado pelo incremento considerável na parcela populacional de idosos, assim como a redução proporcional dos indivíduos nos extratos de idades inferiores constatação reiteradamente verificada em projeções estatísticas em nível global, que somado a melhoria nas condições sanitárias e os novos e variados hábitos, tem resultado no aumento gradual na expectativa de vida de pessoas e grupos (ARAÚJO; POÚL; MARTINS, 2011; BORGES; CAMPOS; SILVA, 2015).

No Brasil, contudo, há de se destacar que o fenômeno em tela, e, por conseguinte, o aumento no quantitativo populacional de idosos, é sui generis, porquanto diferentemente do que ocorreu em alguns países desenvolvidos, os quais foram os primeiros a experimentar alterações demográficas que os fizeram alcançar a transição demográfica de modo lento e gradual, levando mais de um século para chegar à sua plenitude, naquele esse processo tem ocorrido de forma 
acelerada e em curto período, sem que haja tempo suficiente para uma adequada reorganização social (BORGES; CAMPOS; SILVA, 2015; MIRANDA; MENDES; SILVA, 2016).

Nessa perspectiva, portanto, vê-se a partir da análise temporal da demografia brasileira, que em uma década, entre os anos de 1997 a 2007, a população de um modo geral apresentou crescimento relativo da ordem de $21,6 \%$, e, quando analisado o extrato de idosos, os percentuais representam mais que o dobro, devido ao aumento de aproximadamente $47,8 \%$ (BRASIL, 2008a). Isso posto, e, atualmente, com mais de 20 milhões de pessoas com 60 anos ou mais, o país acompanha a tendência mundial ao apresentar uma perspectiva de crescimento deste grupamento populacional para as próximas décadas, devendo atingir 41,5 milhões, em 2030, e 73,5 milhões, em 2060 (BRASIL, 2010a, 2014a; BORGES; CAMPOS; SILVA, 2015).

Não obstante as impressionantes estimativas, para a realidade brasileira, além da diferença na distribuição entre os sexos, cuja razão é de aproximadamente 0.8 , indicando a existência de cerca de 80 homens para cada 100 mulheres, verifica-se, também, diferenciais em relação à colocação dos idosos no território, bem como uma profunda desigualdade social (BORGES; CAMPOS; SILVA, 2015). Ao tempo em que regiões mais desenvolvidas, como o Sudeste, a qual concentram cerca de $80 \%$ do Produto Interno Bruto (PIB), apresentam maior número destes indivíduos, existem aquelas que, embora menos desenvolvidas, tal como o Nordeste, igualmente tem alta concentração, muito provavelmente pela emigração de jovens e adultos, desencadeada pela procura de melhores condições de vida (BORGES; CAMPOS; SILVA, 2015).

Pelo exposto, a despeito da realidade demográfica do Brasil, coloca-se este país em posição de destaque, não apenas pelo conjunto de processos que tem culminado com um aumento cada vez maior de idosos, de forma acelerada, conforme referido, mas também devido à conjuntura em que se insere, sobretudo de desigualdade territorial e social; além de uma série de fragilidades em vários setores, levando-se a crer que, embora considerado um país envelhecido, atualmente, não está adequadamente preparado para lidar com essa realidade, sobretudo no âmbito da Saúde Pública, conforme será mais apropriadamente abordado na seção seguinte (MIRANDA; MENDES; SILVA, 2016).

\section{Elevação da população de idosos e sua repercussão na Saúde Pública}

Não obstante ao que ora foi exposto, a despeito do aumento no contingente populacional de idosos, não se pode crer que esta realidade represente, em si, um problema, sobretudo porque pode ser um indicador indireto do nível de desenvolvimento de uma nação. Mas, em vista de suas implicações em vários setores da sociedade, sobretudo Previdência Social e Saúde Pública, áreas centrais, mas que hoje desafiam países, entre os quais se inclui o Brasil, faz-se mister a consideração deste fenômeno, a fim de que ele possa adquirir relevância no debate público e, mais ainda, no âmbito de efetivação de práticas (BORGES; CAMPOS; SILVA, 2015; MIRANDA; MENDES; SILVA, 2016; PIUVEZAM, 2016).

No campo da Saúde Pública, o crescimento da população de idosos tem repercutido substancial e sistematicamente, uma vez que para além das evidentes mudanças demográficas, tem influenciado alterações no quadro nosológico, notadamente com aumento das DANT, sobretudo àquelas de natureza crônico-degenerativas. Conforme constatado em pesquisa publicada recentemente, por meio da qual os autores traçaram o perfil de morbimortalidade do idoso, e, segundo análise de dados registrados em mais de uma década, verificou-se o predomínio do grupo de enfermidades retro mencionado - sobretudo as doenças do aparelho circulatório, respiratório e neoplasias - entre as principais causas de adoecimento, morte e internações, o que por sua vez aufere relevância a temática em tela, no âmbito sanitário brasileiro (DANTAS et al., 2017).

Destarte, e considerando ainda, a importante associação existente entre os idosos e a dependência física, que altera sobremaneira a autonomia dessas pessoas, crê-se que eles demandam maior atenção em saúde. Constatação esta que se reflete em um heterogêneo perfil de morbidade - com doenças, incapacidades e sequelas - maior tempo de internação hospitalar, elevada frequência de reinternações, assim como da mais lenta recuperação; aspectos que resultam em uma multiplicidade de impactos para aquelas pessoas, em virtude da fragilização da saúde, também para seus familiares, os quais muitas vezes assumem o cuidado sem preparo adequado, e, no plano institucional, uma fonte de grande demanda de recursos para o SUS, que já tem como grande entrave a questão do financiamento (ARAÚJO; PAÚL; MARTINS, 2011; COURA et al., 2015; DANTAS et al., 2017; MIRANDA; MENDES; SILVA, 2016; MIYATA et al., 2005; VIEIRA, 2016).

Ademais, importante considerar que, além do aumento substancioso nos indicadores das DANT em idosos, e as situações que as circunscrevem, conforme outrora colocado, esses indivíduos ainda se deparam com um conjunto de realidades, decorrentes de sua condição e inserção específica na sociedade, as quais, apesar de não se constituírem propriamente adoecimento, contribuem para o sofrimento e também afetam a Saúde Púbica, como os conflitos intergeracionais e a solidão, percebidos em muitos deles. Apesar disso, na contramão dos países considerados econômica e socialmente prósperos, os quais, aliados a patamares elevados no contingente populacional daqueles indivíduos, prepararam-se, do ponto de vista estrutural para lidar com as novas e desafiadoras demandas que surgiram, o Brasil, tal como mencionado, tem apresentado um contínuo e acelerado processo de envelhecimento populacional, sem, no entanto, ter alcançado uma adequada estruturação, sobretudo do ponto de vista da atenção à saúde (BORGES; CAMPOS; SILVA, 2015; MIRANDA; MENDES; SILVA, 2016).

Nesse contexto, no âmbito da Saúde Pública, quer do ponto de vista demográfico, com alteração da estrutura etária, quer do epidemiológico, com o perfil de doenças, internações e incapacidades, o crescimento do número de idosos impele ao sistema de serviços de saúde, tal como se descreve na literatura, uma organização contínua e multidisciplinar, e, particularmente da APS, que pelo caráter central que deveria ter na promoção e prevenção em saúde, tratamento e controle de enfermidades, não apenas aos mais velhos, mas a toda população, vê-se desafiada a fortalecer sua atenção, diante do quadro ora apresentado (MIRANDA; MENDES; SILVA, 2016; OLIVEIRA, 2013; PIUVEZAM, 2016).

Atenção Primária à Saúde e o fortalecimento da assistên- 


\section{cia em saúde do idoso}

A APS, cuja conformação, para a realidade brasileira, tem se estruturado com a Estratégia Saúde da Família, a qual se é creditado um maior potencial para melhoria de saúde das populações, sobretudo para os idosos, indivíduos que cada vez mais têm demandado atenção, mas que podem encontrar naquela um potencial para respostas a até $80 \%$ das necessidades que os levam a procurar um serviço de saúde, por meio de um escopo abrangente de ações que, quando bem estruturadas, contemplam além da promoção e prevenção em saúde, também o tratamento, controle e reabilitação para indivíduos com as mais variadas patologias (CARNEIRO, 2014; GIOVANELLA; MENDONÇA, 2012; ONOCKO-CAMPOS et al., 2014; STARFIELD, 2002).

Destarte, para os idosos, particularmente, a conformação da APS com a abrangência ora exposta, com amplo espectro de atuação é sobremaneira importante, uma vez que, embora nesse grupo imperem uma série de condições que não se restringem ao adoecimento, muito embora contribuam para tanto, as DANT, e as condições que as circunscrevem, de fato, representam uma importante demanda daqueles indivíduos por atenção, as quais requerem, invariavelmente, um cuidado integral, aspecto essencial no manejo de múltiplas e variadas morbidades, bem como na prevenção dos seus respectivos fatores de risco, e, mais ainda, na melhoria da qualidade de vida (GIOVANELLA; MENDONÇA, 2012; MIRANDA; MENDES; SILVA, 2016; DANTAS et al., 2017).

Nesse contexto, sobre as ações de cuidado da APS prestadas aos idosos, estima-se que, aproximadamente, mais da metade da atividade total do profissional de família e comunidade e das prescrições são direcionadas aqueles indivíduos, sendo que a quase totalidade para tratamento ou prevenção das DANT. As características dessas doenças, principalmente a cronificação, impelem a necessidade da continuidade da atenção ofertada, o que pode proporcionar múltiplas e variadas oportunidades para o empreendimento de intervenções efetivas, quer para reduzir a morbimortalidade, quer para melhorar a qualidade de vida, principalmente, uma vez que o aumento da quantidade de anos vividos só pode ser considerado uma conquista, quando acompanhado deste importante fator (VERAS, 2009; OLIVEIRA, 2013).

Ademais, para além da continuidade do cuidado aos idosos, conforme mencionado, também urge a necessidade que se consolide uma instância gestora de cuidados, ou seja, de coordenação, um imperativo na atualidade, uma vez que, em razão da multiplicidade de condições que aqueles são acometidos, não somente, mas sobretudo pelas DANT, conforme colocado, é cada vez mais comum que estes utilizem simultaneamente estruturas de vários serviços do SUS e de diferentes complexidades. Esse fato termina por gerar interdependência entre essas estruturas, carecendo de um reforço para ações de referência e contrarreferência, realidade que, acredita-se, só pode ser plenamente possível se houver, de fato, uma instância que possa coordenar os cuidados prestados, papel que, em tese, deve ser atributo essencial da APS (GIOVANELLA; MENDONÇA, 2012).

Nesse contexto, conforme se defende no presente estudo, importante referência endossa o argumento sobre a importância da coordenação dos cuidados pela APS, em especial aos idosos, na medida em que coloca a ação em tela como indispensável nos dias atuais, principalmente em fun- ção do envelhecimento humano, que tem impelido modificações no panorama epidemiológico, com crescente prevalência das DANT, sobretudo de natureza crônica; além de colocar em evidência que a ação coordenadora proporciona um melhor acompanhamento, sobretudo daqueles indivíduos com múltiplas morbidades, o que é característica dos mais velhos; embora se acredite que os sistemas de saúde na maioria dos países estão, em geral, pouco preparados para tanto (GIOVANELLA; MENDONÇA, 2012).

Nesse contexto, considerando, de um lado, que os idosos, comparativamente a outros grupos de adultos, são mais afetados por múltiplas e variadas morbidades, principalmente as DANT de natureza crônica, e do outro, que em geral demandam cuidados de estruturas e serviços diversos, no âmbito do SUS, é necessário, para bem prestar a atenção almejada, e, principalmente, estabelecer-se enquanto instância coordenadora, o empreendimento de esforços para que a informação seja adequadamente gerida, o que inclui mecanismos adequados de comunicação entre seus profissionais e os especialistas de outros níveis de atenção, fortalecendo a referência e a contrarreferência, portanto; além do adequado registro em prontuários, acessíveis e utilizáveis por todos aqueles responsáveis por cuidados (DANTAS et al., 2017; GIOVANELLA; MENDONÇA, 2012).

Não obstante ao que ora foi exposto, desde a difusão internacional do conceito abrangente e integral creditado à APS, na declaração final dos trabalhos realizados em Alma-Ata, por ocasião da Conferência Internacional sobre Cuidados Primários em Saúde, realizada em 1978, tem-se constatado, não raras vezes, por influência de movimentos ideológicos difundidos por organismos internacionais, sua implementação com conformações variadas: de um lado, abrangente e integral, com importante caráter reorganizador dos sistemas de saúde, e, do outro, seletiva e limitada, sobretudo em países periféricos e outros em desenvolvimento, com arranjos organizacionais orientados pela contenção de gastos e racionalização da assistência individual, passando a designar um conjunto de ações simplórias, com limitados recursos, para poucos agravos e grupos populacionais específicos, sendo operacionalizada por pessoal com baixa qualificação (MEDINA; AQUINO, 2002; PELLEGRINI-FILHO; BUSS; ESPERIDIÃO, 2014).

No âmbito brasileiro, os arranjos de uma APS abrangente e integral, defendida ao longo dos anos pelo movimento sanitário, apesar de conquistada no plano legal, no campo da prática, porém, em não raras realidades, caracteriza-se de modo seletivo e limitado. Isso faz com que ela acumule uma série de problemáticas que ainda dificultam a sua função central de reordenar o SUS e, particularmente, de cuidar das pessoas, ai estando incluídos, necessariamente, os idosos, cujo cuidado é sobremaneira prejudicado por questões que envolvem a gestão do cuidado, fragmentação de ações, o foco em serviços curativos para condições agudas, centralidade no médico, ênfase nas demandas e não exatamente nas reais necessidades dos indivíduos, além do grau de trabalho precário e financiamento insuficiente (GIOVANELLA; MENDONÇA, 2012; ONOCKO-CAMPOS et al., 2014).

Apesar disso, foram ainda implementadas uma série de iniciativas para aumentar e qualificar a APS, dentre as quais se destaca o Pacto pela Saúde, a Política Nacional da Atenção Básica (PNAB), o Programa de Expansão e Conso- 
lidação da Saúde da Família (PROESF), o Programa Nacional de Melhoria do Acesso e da Qualidade na Atenção Básica (PMAQ-AB), o Programa de Valorização dos Profissionais da Atenção Básica (PROVAB) e, mais recentemente, o Projeto Mais Médicos para o Brasil, que tem revolucionado a assistência em cuidados primários em saúde, com provimento emergencial de médicos e fixação destes na atenção primária, reestruturação das unidades básicas de saúde e, ainda, ordenamento da formação médica, de modo a contemplar as necessidades do SUS, sobretudo em cuidados primários (BRASIL, 2003b; 2011a; 2011b; 2013; SANTOS; COSTA; GIRARDI, 2011).

Não obstante, o contexto geral e de limitação da APS, acredita-se que ela vem acumulando uma série de desafios que ainda precisam ser superados, visando à melhoria das condições de saúde das populações, inclusive e talvez principalmente a de idosos, os quais, em sua quase totalidade, dependem exclusivamente dos serviços públicos; situação que tende a se agravar com o aumento exponencial que este grupo tem experimentado nas últimas décadas, e, como já fora reiteradamente colocado, a crescente demanda por cuidados em saúde que estes tem apresentado e que certamente aumentará nos próximos anos (VERAS, 2009; LIMA-COSTA et al., 2011; WITT, 2014). Importa, entretanto, seja qual for a estratégia adotada para a superação das limitações ora colocadas, não prescindir da implementação de mecanismos constantes de avaliação, para que a atenção ofertada seja permanente e criteriosamente observada, de modo a permitir a construção de sólidos indicadores.

Avaliar a atenção primária a saúde para qualificar a assistência em saúde do idoso

A avaliação em saúde na APS, para a realidade brasileira, ganhou força na década passada, na qual as discussões sobre o tema foram ampliadas, reconhecendo a importância do debate a esse respeito, para a construção das políticas públicas, assim como a manutenção das já existentes, instituindo-a enquanto ação de governo e entendendo que esta daria suporte aos processos decisórios do Ministério da Saúde; estando intimamente relacionada à criação da Coordenação de Acompanhamento e Avaliação (CAA), no âmbito do Departamento de Atenção Básica (DAB), da Secretaria de Políticas de Saúde, sendo uma evolução da Coordenação de Saúde da Comunidade, vinculada a então Secretaria de Assistência a Saúde, do Ministério da Saúde (BRASIL, 2005).

Com o desenvolvimento da APS no país, com significativa expansão de sua cobertura, assim como da complexidade da atenção ofertada, em grande parte devido à consolidação das equipes de ESF, enquanto operacionalizador deste nível de atenção, e, em um plano mais geral, como reorganizadora do SUS, ela foi colocada no foco das discussões sobre a ampliação de estratégias para elevar a qualificação das equipes de saúde, tornando suas práticas mais resolutivas, sendo identificada, naquele momento, a necessidade de articulação deste nível de atenção com os demais; e, nesse contexto, as atividades de avaliação desempenhadas pela CAA, bem como do DAB, favoreceram o estabelecimento de uma cultura favorável a tais propósitos (BRASIL, 2005; SOUZA, 2002).

Atualmente, o PMAQ figura como importante ação do Estado, para incentivar os gestores a melhorar a qualidade da assistência ofertada, por meio de ações que contemplam além de estratégias de qualificação e acompanhamento, também aquelas referentes à avaliação do trabalho das equipes de saúde; elevando o percentual de recursos destinados aos municípios que aperfeiçoarem o atendimento geral ofertado; condicionando, portanto, pela primeira vez, o repasse de recursos à implantação e alcance de padrões de acesso e de qualidade pelas equipes, avaliados por parâmetros e indicadores, sobre vários contextos da atenção (PINTO; SOUSA; FLORÊNCIO, 2012; BRASIL, 2014a).

Conforme se apreende da literatura, a formatação e implantação do PMAQ representou um processo de radicalização da lógica de repasses de recursos à APS, uma vez que provoca mudanças substanciosas que levam em consideração importantes indicadores de qualidade, anunciando, inclusive, modificações semelhantes em todo o SUS (PINTO; SOUSA; FLORÊNCIO, 2012). A importância do programa, contudo, não se restringe a mudanças em padrões de financiamento, embora esta seja uma importante contribuição, mas vai além, na medida em que se conforma como uma estratégia para induzir modificações, também, nas condições e formas de funcionamento das próprias equipes de saúde, por meio de objetivos que contemplam parâmetros de comparação; processo contínuo de melhoramento, mobilização de gestores nas três esferas, gestão baseada em resultados, e, principalmente, seu caráter voluntário, que credita aos próprios atores envolvidos, gestores e profissionais, a motivação e proatividade para o seu êxito (BRASIL, 2011; PINTO; SOUSA; FLORÊNCIO, 2012).

De todo modo, apesar do esforço, compreende-se que instituir a avaliação como ação do governo, para nortear a tomada de decisão no âmbito do planejamento e gestão do SUS, constitui-se em um dos seus mais importantes desafios na atualidade. Isso porque ela deve ser sistematicamente integrada aos processos e não de forma incipiente. Para tanto, requer o enfrentamento de, pelo menos, três barreiras, a saber: Técnica, relacionada à definição de critérios, indicadores e a padronização de instrumentos; Cultural-organizacional, com ênfase, portanto, na forma como as instituições desenvolvem suas práticas avaliativas; e, por fim, Políticos, referente às relações de poder entre os atores envolvidos nas políticas de saúde e as esferas de gestão que os avaliam (MEDINA; AQUINO, 2002).

Não obstante a isso, crê se que a cultura de avaliação, dentro do SUS, e, muito particularmente, na APS, se tornará cada vez mais necessária, quer para qualificar a gestão em um cenário de pessimismo econômico que contará com limitados recursos nos próximos anos, quer para o estabelecimento de padrões de qualidade para a atenção; sobretudo em um contexto que, de acordo com as estatísticas oficiais, ratificadas por numerosos estudos, dão conta de um crescimento contínuo e acelerado de idosos, os quais tem demandado cada vez mais assistência, em virtude de variadas enfermidades, não raras vezes múltiplas em um mesmo indivíduo, e, ainda, em grande parte de caráter crônico, o que demanda mais recursos, atenção de qualidade e padrões que certamente podem ser melhor verificados quando da consolidação de uma ampla política de avaliação.

\section{Considerações Finais}


O aumento da expectativa de vida que tem levado, em última instância, à elevação progressiva e acelerada do quantitativo populacional de idosos, quer em países desenvolvidos ou em desenvolvimento, tais como o Brasil, é uma realidade premente, que deve ser encarada com a seriedade que impõe, sobretudo no setor saúde, um dos mais fortemente impactados.

Isso porque o cenário de elevação no contingente de idosos, em relação às populações mais jovens, tem contribuído com a elevação das Doenças e Agravos Não Transmissíveis, sobretudo aquelas de natureza crônico degenerativas, mais comuns entre os mais velhos, em virtude de uma multiplicidade de fatores, necessitando, em um plano mais geral, de acompanhamento durante toda a vida, e, ainda, objetivamente, em não raros casos, maior demanda de atendimentos em diversos pontos de atenção, de distintas complexidades, de internações e medicações de uso contínuo, para citar alguns exemplos; impactando fortemente sistema de saúde, sobretudo o seu financiamento, questão ainda problemática no âmbito do SUS.

Nesse contexto, a atenção primária, então, figura como um cenário privilegiado para dar resposta à crescente demanda dos idosos por atenção em saúde, pela sua capacidade de, quando bem estruturada, ofertar cuidados orientados para a maioria das necessidades e problemas que levam alguém a procurar assistência, e, principalmente, para reorientar o sistema de saúde à promoção em saúde e prevenção de doenças e agravos. Não apenas por ser uma importante estratégia de otimizar os recursos, mas, sobretudo, pela sua capacidade de melhorar a vida das pessoas e grupos, deve ser continuamente aperfeiçoada, para que se alcance a sua consolidação.

Para tanto, defende-se que se fortaleça a cultura avaliativa no âmbito da atenção primária, em evidência, sobretudo após a implementação do Programa Nacional de Melhoria do Acesso e da Qualidade na Atenção Básica, que deve oferecer subsídios para verificar, com critérios, a adequação deste nível de atenção as necessidades reais da população, incluindo os idosos, sobretudo do ponto de vista da estruturação das unidades de saúde e serviços ofertados, formação profissional dos trabalhadores e, por fim, a qualidade e impacto da atenção, propriamente dita.

\section{Referências}

ARAÚJO, I.; PAÚL, C.; MARTINS, M. Viver com mais idade em contexto familiar: dependência no auto cuidado. Rev. Esc. Enf. USP, v. 45, n.4, p. 869-875, 2011.

BORGES, M. G.; CAMPOS, M. B.; SILVA, L. G. C. Transição da estrutura etária no Brasil: oportunidades e desafios para as próximas décadas. In: ERVATTI, L. G.; BORGES, G. M.; JARDIM, A. P (Orgs.). Mudança demográfica no Brasil no início do século XXI: Subsídios para as projeções das populações. IBGE: Brasília, 2015. Disponível em: < http://biblioteca.ibge.gov.br/visualizacao/ livros/liv93322.pdf $>$ Acesso em: 13 Out 2016.

BRASIL. Ministério da Saúde. Projeto de Expansão e Consolidação do Saúde da Família - Proesf. 2003b. Disponível em: <http://bvsms.saude.gov.br/bvs/publicacoes/
PROESF.pdf $>$ Acesso em: 13 Out 2015.

Ministério da Saúde. Avaliação na atenção básica em saúde: caminhos da institucionalização. Brasília, 2005. Disponível em: http://189.28.128.100/dab/docs/publicacoes/ geral/avaliacao_ab_portugues.pdf $>$ Acesso em: 13 Out 2015

Instituto brasileiro de geografia e estatística. Síntese de indicadores sociais 2008 - uma análise das condições de vida da população brasileira. Rio de Janeiro, 2008a. Disponível em: < https://www.nescon.medicina. ufmg.br/biblioteca/imagem/2046.pdf $>$ Acesso em: 13 Out 2015

Instituto brasileiro de geografia e estatística. Sínopse do senso demográfico. 2010a. Disponível em: $<$ http://www.censo2010.ibge.gov.br/sinopse/index. php?dados $=12 \& u f=00>$ Acesso em: 13 Out 2015.

Índice de desempenho do sistema único de saúde. 2011a. Disponível em: $<$ http://idsus.saude.gov.br/mapas. html $>$ Acesso em: 13 Out 2015.

Ministério da Saúde. Portaria 1.654 de 19 de julho de 2011. Dispõe sobre o Programa Nacional de Melhoria do Acesso e da Qualidade. 2011b. Disponível em: <http://bvsms.saude.gov.br/bvs/saudelegis/gm/2011/ prt1654_19_07_2011.html > Acesso em: 13 Out 2015

Ministério da Saúde. Ministério da Educação. Portaria 1.654 de 19 de julho de 2013. Dispõe sobre implementação do Projeto Mais Médicos para o Brasil. 2013c. Disponível em: < http://bvsms.saude.gov.br/bvs/ saudelegis/gm/2013/pri1369_08_07_2013.html > Acesso em: 13 Out 2015

Instituto brasileiro de geografia e estatística. Síntese de indicadores sociais 2014 - uma análise das condições de vida da população brasileira. Rio de Janeiro, 2014a. Disponível em: <http://biblioteca.ibge.gov.br/ visualizacao/livros/liv91983.pdf> Acesso em: 13 Out 2015.

CARNEIRO, T.S.G. O Pacto pela Saúde na prática cotidiana da Atenção Primária à Saúde. Saúde em debate, v. 38, n. 102, p. 429-439, 2014.

COURA, A. S. et al. Quality of life of caregivers of octogenarians: a study using the WHOQOL-BREF. Invest Educ Enferm. v. 33, n. 3, p. 529-538, 2015.

DANTAS, I.C. et al. Perfil de morbimortalidade e os desafios para a atenção domiciliar do idoso brasileiro. Revista Kairós Gerontologia, v. 20, n. 1, p. 93-108, 2017.

GIOVANELLA, L.; MENDONÇA, M. H. M. Atenção primária à saúde: seletiva ou coordenadora de cuidados? Rio de Janeiro (RJ): Cebes; 2012.

LIMA-COSTA, M. F. et al. Tendências em dez anos das condições de saúde de idosos brasileiros: evidências da Pesquisa Nacional por Amostra de Domicílios (1998, 2003, 
2008). Cienc. Saude Colet., v. 16, n. 9, 2011.

MEDINA, M. G.; AQUINO, R. Avaliando o Programa de Saúde da família. In: SOUSA, M. F. (org). Os sinais vermelhos do PSF. São Paulo: Hucitec; 2002. p.135-151.

MIRANDA, G. M. D.; MENDES, A. C. G.; SILVA, A. L. A. Population aging in Brazil: current and future social challenges and consequences. Rev. bras. geriatr. gerontol., v.19, n.3, p. 507-519, 2016.

MIYATA, D. F. et al. Políticas e programas na atenção à saúde do idoso: Um panorama nacional. Arq Cienc Saude Unipar. v. 9, n. 2, p. 135-140, 2005.

OLIVEIRA, E. B. Avaliação da qualidade do cuidado a idosos nos serviços da rede pública de atenção primária à saúde de Porto Alegre, Brasil. Rev. bras. med. fam. comunidade. v. 8, n. 29, p. 264- 273, 2013.

ONOCKO-CAMPOS, R. T. et al. Avaliação da qualidade do acesso na atenção primária de uma grande cidade brasileira na perspectiva dos usuários. Saúde em Debate, v. 38, n. especial, p.252-264, 2014.

PIUVEZAM, G. Atenção primária à saúde e os idosos institucionalizados: a perspectiva da gestão municipal no Brasil. Rev port saúde pública. v. 34, n.1, p. 92-100, 2016.

PELLEGRINI FILHO, A.; BUSS, P. M.; ESPERIDIÃO, M. A. Promoção da Saúde e seus fundamentos: determinantes sociais da saúde, ação intersetorial e políticas saudáveis. In: PAIM, J. S.; ALMEIDA FILHO, N. (Org.). Saúde coletiva: teoria e prática. Rio de Janeiro: Med Book. 2014. p. 305326.

PINTO, H. A.; SOUSA, A.; FLORÊNCIO, A. M. The National Program for Improving the Access and Quality of Primary Care: Reflections on the program design and deployment process. R. Eletr. de Com. Inf. Inov., v. 6, n. 2, 2012.

SANTOS, L. M. P.; COSTA, A. N.; GIRARDI, S. N. Programa Mais Médicos: uma ação efetiva para reduzir iniquidades em saúde. Cienc. Saude Colet., v. 20, n. 11, 2011.

SOUZA, H. M. O PSF como indutor da institucionalização da avaliação na atenção básica. Rev Bras Med Fam Comunidade, v. 6, p. 10-15, 2002.

STARFIELD, B. Atenção primária: equilíbrio entre necessidade de saúde, serviços e tecnologia. Brasília: Ministério da Saúde; 2002. Disponível em: $<$ http://bvsms. saude.gov.br/bvs/publicacoes/atencao_primaria_p1.pdf $>$ Acesso em: 13 Out 2015.

VERAS, R. Envelhecimento populacional contemporâneo: demandas, desafios e inovações. Rev Saúde Pública. v. 43, n. 3, p. 548-554, 2009.
VIEIRA, F. S. Implicações de decisões e discussões recentes para o financiamento do Sistema Único de Saúde. Saúde Debate, v.40, n. 109, p. 187-199, 2016.

WITT, R. R. pois tais pacientes utilizam simultaneamente serviços de diversas complexidades, o que demanda a coordenação entre serviços, função que deve ser exercida pela equipe de atenção primária à saúde. Rev. esc. enferm. USP, v. 48, n. 6, p. 1020-1026, 2014. 